\title{
What gives medical students the edge?
}

\author{
Zhong Jia*, Liangliang Fang ${ }^{\#}$, Yafeng Wan ${ }^{\#}$, Chaojun Kong \\ Department of Hepatobiliary Surgery, Hangzhou First People's Hospital, Nanjing Medical University, PR China
}

${ }^{\#}$ These authors contributed equally to this work

Accepted on January 2, 2018

\section{Letter to the Editor}

Recently, we glimpsed the article entitled as "medical students fall short on blood pressure check challenge" by Abbasi [1] The amazing results reflected the real world of current medical students. Also, the pressing issue has aroused serious concern of the public. Traditional "pure education" seems to be losing its nature of education, which urges to role-shifting via syntegrative education professionals [2]. But what gives medical students the edge?

Based on authors' teaching experience and ongoing work, workplace-based training and "trinity" educational model among medical students have already taken effects with competitive advantages and in prioritized direction. By this way, authors have been attempting to roll out new education model of training [2-4]. The key point to success might be powerfully associated with "syntegrative" education through navigating "pure" education in routine clinical activities. Obviously, it is at a critical juncture when medical students start to play new role in teaching hospital. To best of our knowledge, progress in education reforms relies on three aspects of clinical service, including constant funds support, syntegrative education reform, and avoiding mental disorder or psychological depress [5]. In practice, one-third of successes contribute to "hardware" such as national training bases, while the other two-thirds thank to "software" such as abilities to settle practical problems emerging in workplace. The complex interplay of these sectors will determine whether education reform is successfully addressed or whether medical students can benefit from quality training of professionals by live pilot at promising fields. Online class-sharing, offline multiple channels education, cultivating communication skills in various clinical activities, academic performance, and even can-do spirits, all of these aspects have to be comprehensively considered in this shifting dynamic to better patient-centered health care $[3,4]$.

Undoubtedly, trinity education model provides medical students new ways to get access to professional and social context. Each step will be tested by real-time reflect via interactive question and answer by using chatting tools such as Facebook or Twitter in smartphone. Besides, more importantly, medical trainees will obtain creative thinking, communicative skills and can-do spirits in a long run, not merely pure medical knowledge, which is believed to be an essential precondition to reshape the edge of medical students from its beginning.

As a matter of fact, the nature of education reform is to integrate various resources of education and related knowledge or skills, including general and professional learning, communication skills, perception of clinical problems, humanism care, academic or innovative performance, and even leadership, to develop students' untapped talents for the future medical science.

Provisionally, artificial intelligence and robots are already expected to disrupt and replace many traditional industries in the coming decade. That also means trainees can spend more time on steering the new technologies or solving clinical issues, instead of repeated e-medical records that can occupy and exhaust medical students major energy and time. The syntegrative education also provides medical students more options to filter their interests and passion. Therefore, they can adjust to the system earlier and offer better health care.

Should medical students say goodbye to silos education? The answer is YES because work experience and basic skills will give them the edge.

All in all, authors readmit that trinity education professionals may point out an accessible pathway to sharpen medical students the edge and the randomized control trial of the idea is ongoing.

\section{References}

1. Abbasi J. Medical students fall short on blood pressure check challenge. JAMA 2017; 318: 991-992.

2. Jia Z, Zhang J, Kong CJ. Medical students urged to "trinity"-based professional education transformation. Austra Med J 2017; 10: 904-905.

3. Zhou Y, Jia Z, Kong S. Is the future health care technology or goal-driven? Chin Med J 2017; 130: 1886.

4. Ball DL, Cohen DK. Developing practice, developing practitioners: Toward a practice-based theory of professional education. Teach Learn Profess Handbook Policy Pract 1999; 1: 3-22.

5. Dyrbye LN, Thomas MR, Shanafelt TD. Systematic review of depression, anxiety, and other indicators of psychological distress among US and Canadian medical students. Acad Med 2006; 81: 354-373. 


\section{*Correspondence to}

Jia Zhong

Department of Hepatobiliary Surgery

Hangzhou First People's Hospital

Nanjing Medical University

PR China 